was tested by showing that the thermal stability of isolated UUCG hairpins was substantially greater than several control hairpin sequences. GNRA hairpins are also exceptionally stable (J. Haney, unpublished data).

Cheong et al. ${ }^{1}$ have now determined the high-resolution structure of a 12nucleotide UUCG hairpin using NMR methods originally developed by Wuthrich $^{13}$ for proteins. Instead of relying entirely on interproton distances supplied by nuclear Overhauser enhancement (NOE) build-up rates, estimates for three of the five backbone bond angles were obtained by measuring proton-proton scalar coupling constants. The authors used no less than 395 distance and angle constraints to calculate the final structure using distance geometry and constrained energy minimization methods ${ }^{1}$. The resulting structure is in satisfactory agreement with the phylogenetic, thermodynamic and chemical modification data. Perhaps the greatest surprise is that a syn $\mathrm{G}-\mathrm{U}$ base pair forms between the first and last residues of the loop, effectively making a loop of only two residues. To reverse the direction of the polynucleotide chain, the riboses of the two remaining single-stranded residues have an extended DNA-like C 2 '-endo configuration. The phylogenetically variable and chemically reactive $U$ residue protrudes into solution whereas the $\mathrm{C}$ residue is stacked inside the loop and makes an internal cross-loop hydrogen bond. Presumably this hydrogen bond and the extra base pair are responsible for the exceptional stability of the hairpin.

RNA biochemists have for a long time wanted to use computer folding algorithms to predict RNA secondary structures successfully. Nearly 20 years have passed since Tinoco and colleagues ${ }^{1+}$ delineated a thermodynamic aproach to the RNA folding problem. Essentially, they considered each RNA structure to contain a number of basic thermodynamic elements (helix stacking interactions, loops, bulged nucleotides and so on), each contributing a characteristic amount of free energy (positive or negative) to the overall stability of the molecule independent of the particular RNA in which they occured. The authors then analysed the melting profiles of small model RNA olignucleotides to deduce values of free energy for each element (the 'Tinoco rules'). Possible combinations of base-paired regions in a given RNA sequence were identified by computer and the total free energy calculated for each. The structure with the lowest free energy was then assumed to be the correct one.

Despite considerable improvements in the programs that find possible structures ${ }^{15}$ and a steadily increasing library of model compounds ${ }^{16}$, it is clear that the method is not yet able to predict the structure of longer RNA molecules reliably. For example, several of the current computer algorithms did not successfully predict the secondary structure of Bacillus subtilis P RNA deduced by phylogenetic methods? The basic problem is that many elements that either stabilize or destabilize RNA structure remain to be identified. These include long-range base-pairing interactions such as pseudoknots $^{17}$, triple-stranded tertiary interactions of the type found in TRNA and, of course, unusual loop structures exemplified by the UNCG and GNRA tetraloops. Until these additional elements are identified and their thermodynamic contributions measured, the predicted structures will contain errors.

How much does the newly acquired knowledge of the thermodynamics of the UNCG and GNRA tetraloops aid in predicting secondary structure? An answer is provided by Jaeger et al. ${ }^{18}$ who assumed an extra $2 \mathrm{kcal} \mathrm{mol}^{-1}$ for each of these structures and found a small but consistent improvement in their ability to predict phylogenetically proven RNA structures. Perhaps the experience with RNA tetraloops points the way to a synthesis of the more biological (phylogenetic) and chemical (thermodynamic) approaches to RNA structure. Chemists can solve the structures and measure the thermodynamic properties of units of RNA identified by biologists. The resulting more accurate folding algorithms will be invaluable for molecular biologists interested in the structures of mutant RNAs that cannot be solved by phylogenetic methods.

Olke C. Uhlenbeck is in the Department of Chemistry and Biochemistry, University of Colorado, Campus Box 215, Boulder, Colorado 80309, USA.

. Cheong, C., Varani, G. \& Tinoco, I. Nature 346, 680 $682(1990)$

2. Groebe, D. R. \& Unlenbeck, O. C. Nucleic Acids Res. 16 11725-11375 (1988)

3. Haasnoot, C.A.G., Hilbers, C.W., van der Marel, G.A. \& van Boom, J.H. J. Biosci. 8, 767-780 (1985).

4. Woese, C. R., Gutell, R., Gupta, R. \& Noller. H.F. Microbiol. Rev. 47, 621-669.

5. Guttel, R. R. \& Fox, G. E. Nucleic Acids Res. 16 (suppl.), 175-r269 (1988)

6. Moazed, D., Stern, A. \& Noller, H.F. J. molec. Biol. 187 399-416 (1986)

7. Siegal, V. \& Walter, P. Proc. natn. Acad. Sci. U.S.A. 85 1801-1805 (1988)

8. Liao, X., Brennwald, P. \& Wise, J.A. Proc. natn. Acad Sci. U.S.A. 86, 4137-4141 (1989)

9. Pace, N. R. Smith, D.K., Olsen, G.J. \& James, B.D. Gene 82, 65-75 (1989)

10. Waring, R. B. \& Davis, R. W. Gene 28, 277-291 (1984).

11. Jaquier, A. \& Michel, F. Cel/50, 17-29 (1987).

12. Tuerk, C. et al. Proc. natn. Acad. Sci. U.S.A. 85, 1364 1368 (1968).

13. Wuthrich, K. in NMR of Proteins and Nucleic Acids (Wiley, New York, 1986)

14. Tinoco, i., Uhlenbeck, O.C. \& Levine, M.D. Nature 230, 362-367 (1971)

15. Zuker, M. Science 244, 48-52 (1989)

16. Turner, D. H. \& Sugimoto, N. A. Rev. Biophys, biophys. Chem. 167-192 (1988)

17. Pieij, C. W. A., Rietveld, K. \& Bosch, L. Nucleic Acids Res 13, 1717-1731 (1985)

18. Jaeger, J. A., Turner, D.H. \& Zuker, M. Proc. natn. Acad. Sci. U.S.A. 86, 7706-7710 (1989)

\section{Currents of air}

MODERN windmills, while a great improvement on their traditional ancestors, are still big and expensive monsters. They disfigure any landscape, and can cause television reception to flutter wildly. Daedalus now has a new approach.

The heated cathode of a radio valve is a copious emitter of electrons. What would happen, he wonders, if they were emitted not into a vacuum but into the air? They would immediately latch onto air molecules to form negative ions. Unlike electrons, ions can diffuse only slowly through the surrounding air. A wind blowing past the cathode would carry them away bodily, and could even 'pump' them against a considerable electric field. Quite a small valve-cathode can eject an amp of electrons, so a big mesh emitter erected on a windy site could launch a heavy current of ions. The wind would drive them to a parallel collecting mesh a few centimetres away. If the two electrodes were connected by a cable, the resulting return current would capture much of the energy of the wind blowing through the mesh.

This neat 'ionic windmill' has an obvious snag. The heated cathode of an evacuated valve consumes only a small fraction of the power conducted by the valve; but a large wind-blown heated emitter might well need almost all its captured power to keep itself hot. To make an energetic profit, the emitter may have to work in the cold. So Daedalus now intends to equip it with many small sharp discharge points, coated with materials of the lowest possible work function. He will charge the entire circuit to such a high negative voltage that the emitter grid sprays out ions copiously even at room temperature. Blown downwind to the rounded wires of the nearby collector grid, they will entrap wind energy with no needless thermal losses.

Even the best cold mesh emitter is unlikely to ionize more than about one in ten million of the air molecules blowing through it. A strong breeze might release a current of a few amps per square metre, and be able to blow it up a potential difference of 100 volts or so. Accordingly, a 100metre-square ionic windmill could generate several megawatts of useful power.

Silent and unobstrusive, the ionic windmill will revolutionize the renewable energy business. Modern inverters and control electronics could easily convert its fluctuating output to a stable standard line voltage. It need not even rotate to face the wind; a static array in star or delta form could generate power in the most wildly veering breeze. It would be no more hazardous than any other high-voltage installation. And the inevitable leakage of negative ions into the air should subtly cheer up people living downwind.

David Jones 